\title{
Puerto Rico Health System Resilience After Hurricane Maria: Implications for Disaster Preparedness in the COVID-19 Era
}

\author{
Christopher C. Rios ${ }^{1}$, Emilia J. Ling ${ }^{1}$, Ralph Rivera-Gutierrez ${ }^{2}$, Juan Gonzalez Sanchez ${ }^{3}$, \\ Sylvia Bereknyei Merrell ${ }^{4}$, Janine Bruce ${ }^{5}$, Michele Barry ${ }^{6}$ and Vinicio de Jesus Perez ${ }^{7 *}$ \\ ${ }^{1}$ Stanford University School of Medicine, Stanford, CA, United States, ${ }^{2}$ School of Public Health, University of Puerto Rico \\ Medical Science Campus, Rio Piedras, PR, United States, ${ }^{3}$ Department of Emergency Medicine, University of Puerto Rico \\ Medical Science Campus, Rio Piedras, PR, United States, ${ }^{4}$ Department of Pediatrics, S-SPIRE Center, Stanford University \\ School of Medicine, Stanford, CA, United States, ${ }^{5}$ Department of Pediatrics, Stanford University School of Medicine, \\ Stanford, CA, United States, ${ }^{6}$ Department of Internal Medicine, Stanford University School of Medicine, Stanford, CA, \\ United States, ${ }^{7}$ Division of Pulmonary Allergy and Critical Care Medicine, Stanford University Medical Center, Stanford, CA, \\ United States
}

\section{OPEN ACCESS}

Edited by:

Sumana Chattopadhyay, Marquette University, United States

Reviewed by:

Fantina Maria Santos Tedim, University of Porto, Portugal John James Kiefer,

University of New Orleans,

United States

${ }^{*}$ Correspondence:

Vinicio de Jesus Perez

vdejesus@stanford.edu orcid.org/0000-0001-5532-8247

Specialty section:

This article was submitted to Disaster Communications, a section of the journal Frontiers in Communication

Received: 11 August 2020 Accepted: 27 November 2020 Published: 03 February 2021

Citation:

Rios CC, Ling EJ, Rivera-Gutierrez R, Gonzalez Sanchez J, Merrell SB, Bruce J, Barry M and de Jesus Perez V (2021) Puerto Rico Health System Resilience After Hurricane

Maria: Implications for Disaster Preparedness in the COVID-19 Era.

Front. Commun. 5:593939. doi: 10.3389/fcomm.2020.593939
Background: Every year, Puerto Rico faces a hurricane season fraught with potentially catastrophic structural, emotional and health consequences. In 2017, Puerto Rico was hit by Hurricane Maria, the largest natural disaster to ever affect the island. Several studies have estimated the excess morbidity and mortality following Hurricane Maria in Puerto Rico, yet no study has comprehensively examined the underlying health system weaknesses contributing to the deleterious health outcomes.

Methods: A qualitative case study was conducted to assess the ability of the UPR health system to provide patient care in response to Hurricane Maria. An established five key resilience framework and inductive analysis was used to identify factors that affected health system resilience. Thirteen Emergency Medicine Physicians, Family Medicine Physicians, and Hospital Administrators in a University of Puerto Rico (UPR) Community Hospital were interviewed as part of our study.

Results: Of the five key resiliency components, three domains were notably weak with respect to UPR's resiliency. Prior to the Hurricane, key personnel at the UPR hospital were unaware of the limited capacity of back-up generators at hospitals and were ill-prepared to transfer ICU patients to appropriate hospitals. Post Hurricane, the hospital faced self-regulation challenges when triaging the provision of Hurricane-related emergency services with delivering core health services, in particular for patients with chronic conditions. Finally, during and after the Hurricane, integration of patient care coordination between the UPR hospital ambulances, neighboring hospitals, and national and state government was suboptimal. The two remaining resiliency factors, addressing diverse needs and system adaptiveness in a time of crisis, were seen as strengths.

Conclusions: Hurricane Maria exposed weaknesses in the Puerto Rican health system, notably the lack of awareness about the limited capacity of backup generators, poor patient care coordination, and interruption of medical care for patients with chronic conditions. As in other countries, the current COVID epidemic is taxing the capacity 
of the Puerto Rico health system, which could increase the likelihood of another health system collapse should another hurricane hit the island. Therefore, a resilience framework is a useful tool to help health systems identify areas of improvement in preparation for possible natural disasters.

Keywords: health system resilience, disaster preparedness, resilience framework, COVID-19, Puerto Rico

\section{INTRODUCTION}

In September 2017, Hurricanes Irma and Maria devastated Puerto Rico and caused severe damage to critical infrastructure and essential health systems (Kishore et al., 2018; SantosBurgoa, 2018). This led to an ongoing, massive shortage of electricity and clean water supplies, which had substantial impact on the health and well-being of the Island's population. Although disruption in healthcare services is expected during any natural disaster, health systems must be able to minimize these disruptions and respond to these stressors in a time of need (Kruk et al., 2015, 2017; Ling et al., 2017).

Recently, there has been a growing body of literature characterizing the importance of resilience in health systems. Now, with the spread of COVID-19 challenging the integrity of health systems around the globe, this concept has once again been brought to the forefront. Resilience, defined as the capability of a health system to prepare, respond and reorganize under conditions of stress, is posited to protect the population from excess morbidity and mortality (Greenstein et al., 2016; Kutzin and Sparkes, 2016; Blanchet et al., 2017; Kruk et al., 2017; Ling et al., 2017; Abimbola and Topp, 2018). In this setting, excess mortality and morbidity is defined as an elevation in the number of deaths and/or prevalence of disease in the months to years following the stressor as compared to the expected number of deaths and/or prevalence of disease if the stressor had not occurred. The most comprehensive assessment of Hurricane Maria found that from September 20, 2017 to December 31, 2017, there was an estimated 4,645 excess deaths largely due to delayed or inadequate access to healthcare services (Kishore et al., 2018). Moreover, various studies have shown that people with chronic medical conditions, and persons in the lowest socioeconomic category were disproportionately affected by Hurricane Maria (Kishore et al., 2018; Mattei et al., 2018; Pullen, 2018; Santos-Burgoa, 2018).

Although there has been extensive literature focusing on the definition of resilience in health systems, there have been few studies which apply the resilience framework to health systems ( $\mathrm{Li}$ et al., 2008; Kutzin and Sparkes, 2016). In this study, a qualitative approach is used to bring the resilience framework from the theoretical to the practical. Specifically, a resilience framework is used to identify key factors that may have contributed to excess morbidity and mortality in Puerto Rico following Hurricane Maria.

\section{METHODS}

\section{Setting, Context, and Rationale}

We conducted a case study using qualitative methods, as this approach allowed us to better understand the "why" and "how" of excess mortality and morbidity following Hurricane Maria (Lin, 1993). The UPR Community Hospital was purposefully selected as the "critical case" given its central location in San Juan (the capital of Puerto Rico), because it was one of the few hospitals that remained open throughout Hurricane Maria and the critical weeks following the natural disaster, and it has disaster-preparedness systems similar to other hospitals. By using the critical case purposeful sampling strategy a logical generalization can be made that other hospitals in Puerto Rico and the Puerto Rican health system overall experienced similar challenges. Moreover, the Kruk et al. framework was constructed for both hospitals and health systems, and with the critical case sampling, we can make a logical generalization of the UPR Hospital that may be indicative of the greater Puerto Rican health system. Due to these characteristics, this "critical case" case study about this UPR Community Hospital's resilience may help to identify successful strategies that other hospitals/healthcare systems may consider when implementing policies to address resilience factors (Lin, 1993; Patton, 2002).

\section{Participants and Approach}

To assess hospital and health system resilience, semi-structured in-depth interviews were conducted with key stakeholders at the selected UPR Community Hospital (Table 1). We used key informant sampling of physicians who were on service for at least 3 days in the week before or after Hurricane Maria. To capture a breadth of understanding and experiences, we identified Emergency Medicine (EM) physicians, Family Medicine (FM) physicians, and Administrators involved in delivering or coordinating patient care before, during or after Hurricane Maria. The interviews generally lasted between 30 and $70 \mathrm{~min}$. Reflexivity was maintained by researchers throughout the interview and analysis process by recording, discussing, and challenging established assumptions throughout the data collection and analysis.

An interview guide was created by the first and second author adapted from a similar study conducted in Ebola by the second author (Ling et al., 2017). The interview guide was designed to prompt discussion about the strengths and weaknesses the participants observed after and before the Hurricane through the lens of the five resilience domains. The 


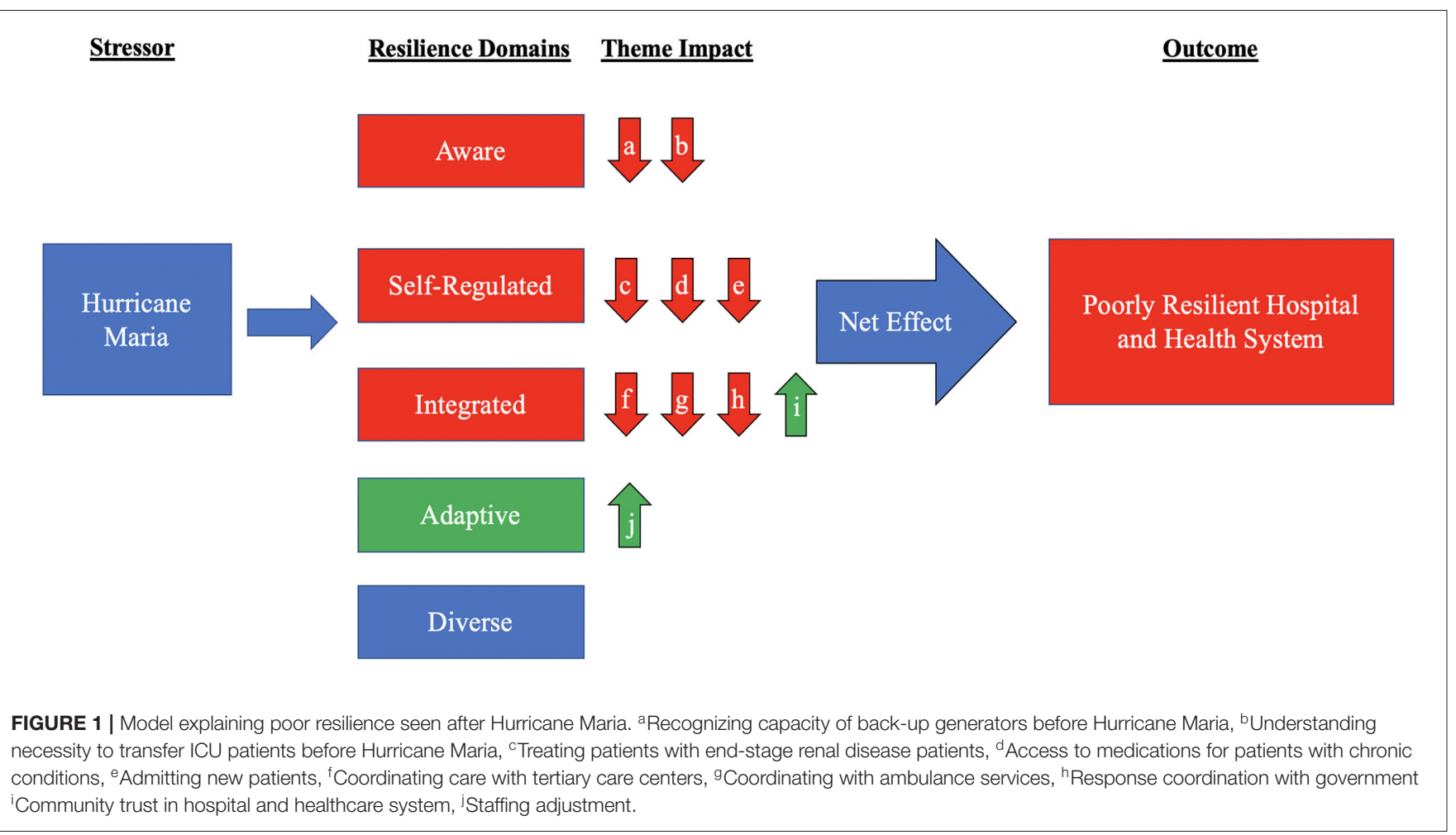

TABLE 1 | Demographics of Respondents.

\begin{tabular}{lcc}
\hline & Subjects $(\boldsymbol{n = 1 3})$ & Percentage \\
\hline Male/Female & $5 / 8$ & $38.5 / 61.5$ \\
Years of experience & 3 & \\
$0-1$ & 6 & 23.1 \\
$1-2$ & 4 & 46.2 \\
$3+$ & & 30.8 \\
Hospital affiliation & 4 & \\
Emergency Medicine (EM) & 4 & 30.8 \\
Family Medicine (FM) & 5 & 30.8 \\
Administration & & 38.5 \\
Time spent at hospital & 9 & \\
1-3 days pre-hurricane & 10 & 75.0 \\
During hurricane & 11 & 83.3 \\
1-3 days post-hurricane & 2 & 91.7 \\
Only after & & 16.7 \\
\hline
\end{tabular}

questions were revised and adapted to the cultural context, then reviewed by two qualitative research experts for integrity. Key questions from the interview guide are presented in Table 2. Interviews were conducted between June 15, 2018 and August 5, 2018. All interviews were conducted in-person with identified stakeholders.

Confidentiality and anonymity were discussed with participants during the consent process. Interviews were audiotaped for transcription and purposes conducted in both
TABLE 2 | Sample Interview Questions by Domain.

\begin{tabular}{ll}
\hline Domain & Question \\
\hline General & $\begin{array}{l}\text { In your opinion, which of these [Resilience] domains } \\
\text { is the most important to ensure the population is } \\
\text { safe during a crisis? } \\
\text { What has the hospital done to obtain and use } \\
\text { information in order to prepare and respond to the } \\
\text { needs of the community during the Hurricane? Can } \\
\text { you give examples? } \\
\text { Before Hurricane Maria, what areas of patient care } \\
\text { were strengths and what were seen as weakness of } \\
\text { patient care at this center? } \\
\text { Diverse } \\
\text { During the initial response to Hurricane Maria, what } \\
\text { was done to address the chronic conditions in the } \\
\text { population? } \\
\text { During the response to Hurricane Maria, how was } \\
\text { the coordination between the government, other } \\
\text { hospitals, and the private sector? } \\
\text { Integrated }\end{array}$ \\
$\begin{array}{l}\text { During the response to Hurricane Maria, what } \\
\text { changes did the hospital make to improve patient } \\
\text { care? }\end{array}$
\end{tabular}

Spanish and English depending on the preference of the participant. All study findings, including quotations, have been appropriately anonymized, and data collected through this study was safely stored in a password-protected laptop. The Stanford Institutional Review Board approved all research procedures as an exempt study. 
TABLE 3 | Health System Resilience Domains.

\begin{tabular}{|c|c|}
\hline Domain & Definition \\
\hline Aware & $\begin{array}{l}\text { Obtains a comprehensive, up-to-the-minute } \\
\text { understanding of the specific health needs of the } \\
\text { population as well as the human, physical, technical } \\
\text { assets necessary to meet those health needs }\end{array}$ \\
\hline Diverse & $\begin{array}{l}\text { Meets a substantial breadth of the population's } \\
\text { health needs }\end{array}$ \\
\hline Self-Regulating & $\begin{array}{l}\text { Continues to meet the basic health needs of the } \\
\text { population when the hospital or system is under } \\
\text { significant stress }\end{array}$ \\
\hline Integrated & $\begin{array}{l}\text { Effectively and efficiently brings together diverse } \\
\text { group of actors to coordinate patient care and } \\
\text { deliver health services }\end{array}$ \\
\hline Adaptive & $\begin{array}{l}\text { Transforms when under stress to meet the specific } \\
\text { health needs of the population }\end{array}$ \\
\hline
\end{tabular}

\section{Data Analysis Using the Resilience Framework}

For our starting domains, we used the Kruk framework of health system and hospital resilience. Under this framework, health system or hospital resilience is broken down into its five foundational components: (1) Aware, (2) Diverse, (3) SelfRegulating, (4) Integrated, and (5) Adaptive (Ling et al., 2017). With respect to Aware, resilient health systems and hospitals have a comprehensive, up-to-the-minute understanding of the specific health needs of the population as well as the human, physical, technical assets necessary to meet those health needs. With respect to Diverse, resilient health systems and hospitals have the ability to meet a substantial breadth of the populations health needs. With respect to Self-Regulating, resilient health systems and hospitals are able to continue meeting the basic health needs of the population when the hospital or system is under significant stress. With respect to Integrated, hospitals and health systems are able to effectively and efficiently bring together diverse groups of actors to coordinate patient care and deliver health services. Finally, with regard to Adaptive, resilient hospitals and health systems are able to transform when under stress to meet the specific health needs of the population. Table 3 summarizes the foundational components and the characteristics of resilient hospitals and health systems.

After using the Kruk resilience framework as initial codes, we conducted iterative inductive analysis to identify additional resilience themes that arose from the data. Inter-rater reliability was achieved via Dedoose training to ensure credibility of the results (Dedoose, 2018). Specific resilience themes were identified through data familiarization by reading and rereading coded excerpts. Through this method, various factors of health system resilience were identified that participants expressed likely contributed to excess morbidity and mortality. Finally, this study fulfills standards for reporting qualitative research (Strauss and Corbin, 1998; O’Brien et al., 2014).

\section{Research Reporting Checklist}

We used the SRQR reporting guideline (O'Brien et al., 2014).

\section{RESULTS}

In the following section, we report on the five resilience framework categories and the inductively derived themes/subthemes that emerged in the context of Hurricane Maria. Representative quotes for each theme/sub-theme are noted in each section and summarized in Table 4.

\section{Aware}

With respect to the Aware component, resilient health systems and hospitals have a comprehensive, up-to-the-minute understanding of the specific health needs of the population as well as the human, physical, technical assets necessary to meet those health needs. Within Aware, we identified two domains that represent resilience weakness of the Puerto Rican health system.

\section{Recognizing Limited Capacity of Emergency Back-Up Generators Before Hurricane Maria}

Physicians and administrators described the lack of back-up generators affecting patient care in a variety of ways, and many described it as the single greatest factor contributing to interruption of patient care. One major problem was the lack of temperature regulation for very ill patients. As one EM physician describes, "I think one issue was the heat. The patients that we weren't able to bring down to floors that were cooler, their family brought them a fan. So, it was really, really hot and, in part, that affected mortality. I feel like the heat was part of it." Another FM physician described the difficulty of treating patients in labor and delivery without power, "It was very hot, and humid-but we tried to maintain the best possible habitat for the pregnant ladies. But there was a point where the labor room-wasn't able to take any more patients, that was an order of the medical director because-it was not okay to work in those conditions." Patients had to be moved from floors without electricity because of the heat, which resulted in significant overcrowding of the emergency room and lobby. To many of the respondents, the power supply was the ultimate limiting factor when it came to providing adequate care, "The power supply was our limitation. We needed a power generator with enough capacity to supply the entire hospital. There was no oxygen, and patients were very hot. If the patient had an ulcer, the ulcer got worse."

\section{Understanding Necessity to Transfer ICU Patients Before Hurricane Maria}

Given the constraints on power supply, this UPR Community Hospital was unable to provide adequate care for patients in the ICU and many of these patients had to be chaotically transferred to other hospitals in the days following the hurricane. One administrator noted, "We need to identify the hospital's vulnerable areas to protect the patient in those areas or evacuate them prior to the disaster." Another administrator noted the difficulty transferring these patients following the Hurricane, "It was difficult to transfer patients to be admitted to other health centers. It wasn't easy to write a memo or call and say, 'I have 20 patients that need to be moved.' In the end we made it, but we need to improve this part." 
TABLE 4 | Hospital/Health System Themes and Sub-themes with Representative Quotes.

Representative quotes

\section{Aware}

Recognizing capacity of back-up generators before Hurricane Maria

Understanding necessity to transfer ICU patients before Hurricane Maria

\section{Self-Regulated}

Treating patients with chronic conditions

Treating patients with end stage renal disease

Access to medication for patients with chronic conditions

Admitting new patients

\author{
Integrated \\ Coordinating care for the \\ critically ill \\ Communication with tertiary care \\ centers
} Coordination with ambulance
services

Response coordination with

Community trust in hospital and health system

Adaptive

Staffing adjustments government

"If they'd had the power plant from the beginning, the hospital would've run without a problem the whole time. There would've been a greater number of patients expected in a crisis, but the problem was the power plant. That made the hospital unable to provide complete service during that period." -ED Physician

"Patients in the intensive care unit, an area that needs improvement, or else, patients will need to be moved before any other crisis." -Administrator

"So diabetic patients, hypertension patients, chronic heart failure patients, were decompensated for not having medication during the first days of the hurricane... so, they would come decomp[ensated]-acute, over chronic conditions." -Internal Medicine Physician

"Puerto Rico has to work with companies that offer dialysis services to maintain a safe structure and guarantee dialysis services to the patients. I dare to say that this could have caused deaths of many patients. They could have been avoided if these companies had a safe structure to provide their services." -Administrator

"They're blood pressure medications. They're diabetes medications. They're depression medication, anxiety medication, things that they were able to refill, that they couldn't refill because everything was closed... Patients who literally would only come in because they has asthma, and they just wanted to treat their asthma... Those were less acute patients that we had to host, and it was difficult situations." -ED Physician

"We couldn't really admit patients into the floors because, like I said, there was no air conditioning, and they were bringing all the people down to lower floors and discharging as many people as they could, some people had nowhere to be discharged to. We couldn't admit too many people at our floor, we had to transfer to the other main hospital." -Family Medicine Physician

"We need to improve communication with other health centers. We think we're prepared, but it's not enough; we need to have bigger support and coordinate better with hospitals. We have to offer our support to other health centers too. The first days were really hard; I have to be honest." -Emergency Medicine Physician

"This is a country with limited resources. I understand we all have limited resources, but we definitely have to improve the interrelation among health centers. We need to develop better communication ways, including communication between hospitals and with the federal government; it has to be a friendly communication system. What matters here is the human being, the patient." -Internal Medicine Physician

"You couldn't coordinate for a patient to be transferred through our ambulance. At the same time, it's like, how are you going to discharge somebody who has an amputation that needs treatment." -Emergency Medicine Physician

"Regarding my perspective on the communication between the hospital and the government... they took too long. The truth is given the crisis at the time, they couldn't take care of all the hospitals. Our hospital is near the airport; our population is old, and they needed to be a priority." -Administrator

"The community trusts this hospital, and they know that even with a big crisis, they can count on the hospital's services because the hospital's door will always be open." -Emergency Medicine Physician

"They did a really good job right after making sure that we had enough staff. We worked doubles. Not double shifts, but we had a double coverage. The nursing staff was really amazing about that. We have people that live really far away. We had one nurse who actually lost her whole house and they were really supportive about getting her new uniforms and helping her get to work. We rallied together. They were really helpful in that sense." -Emergency Medicine Physician

Diverse

None

$\mathrm{N} / \mathrm{a}$

\section{Self-Regulated}

With respect to Self-Regulated component, resilient health systems and hospitals are able to continue meeting the basic health needs of the population when the hospital or system is under significant stress. Within Self-Regulated, we identified two domains, and two sub-domains, as weakness in the resilience of the Puerto Rican health system.

\section{Treating Patients With Chronic Conditions}

The two populations that were most adversely affected were patients with end stage renal disease (ESRD) and patients that needed access to medications for chronic conditions.
These findings corroborate other studies that suggest elderly populations with chronic conditions were disproportionately affected by Hurricane Maria (Mokdad et al., 2005; Kishore et al., 2018; Roman, 2018).

\section{Limited Treatment for Patients With End Stage Renal Disease (ESRD)}

Patients with ESRD rely on regular hemodialysis to avoid lifethreatening metabolic derangements such as hyperkalemia or uremia (Hakim and Lazarus, 1995). Given the prevalence of diabetes in Puerto Rico it is no surprise that there is a significant population with ESRD reliant on regular hemodialysis (Mattei 
et al., 2018). In the weeks following Hurricane Maria, these patients experienced extreme difficulty accessing treatment. As one EM physician stated, "As far as chronic patients, kidney failures were the most frequent visitors to the hospital after the event because they hadn't been able to get their dialysis during those days." Respondents noted that in the days and weeks following Hurricane Maria, decompensated patients with ESRD presented to the emergency department for emergent dialysis, "We had to deal with patients who had to be dialyzed urgently, so I think that having an additional dialysis unit for emergencies to help those patients could be an improvement." In the aforementioned cases, patients were able to ultimately seek medical attention, but it is likely that many other patients with ESRD, especially those living in remote areas, were not able to access care.

\section{Limited Access to Medications for Patients With Chronic Conditions}

Patients with other chronic conditions like COPD, asthma, heart failure and others who also rely on consistent medical management to ensure that they do not decompensate into a lifethreatening state, were disproportionally affected by Hurricane Maria. One administrator noted, "Diabetic patients, hypertension patients, chronic heart failure patients, were decompensated for not having access to mediation during the first few days of the hurricane. So they would come in decompensated-acute over chronic conditions." Another respondent noted the difficulty addressing this situation, "There are no health centers for patients who need this type of long-time treatments. We didn't have the resources because there were not that many beds." Although many of the patients were ultimately able to receive respiratory treatment or access to their medications, many patients in remote regions of Puerto Rico were not able to travel to an emergency department to receive therapy. Moreover, even for patients that did manage to ultimately access their medications, acute exacerbation of chronic conditions often lead to increasing morbidity and more rapid disease progression.

\section{Reduced Admission of New Patients}

Respondents noted that, shortly after Hurricane Maria, the hospital was no longer able to admit new patients. As one EM physician noted, "We couldn't admit patients so the challenge was: should we close our doors to these patients because we can't offer them the services that they most deserve?" This created a chaotic situation where acutely ill patients had to be managed exclusively in the emergency department or sent away. Another EM physician noted, "If a patient with an infected wound, who has to be admitted to the hospital, came in, that patient wouldn't be admitted." Ultimately, these patients would be transferred to another hospital, but the inability to admit represented a significant delay in medical care.

\section{Integrated}

With respect to the Integrated component, hospitals and health systems are able to effectively and efficiently bring together diverse groups of actors to coordinate patient care and deliver health services. Within Integrated, we identified three domains and two sub-domains as weaknesses in the Puerto Rican health system.

\section{Coordinating Care for Critically III}

One of the most significant challenges encountered by the UPR Community Hospital following Hurricane Maria was coordinating care for the critically ill. As one EM physician noted, "The coordination, on the first day, the first days, the first weeks, it was nearly impossible because there was no communication."

\section{Broken Communication With Tertiary Care Centers}

Immediately following Hurricane Maria, many patients had to be transferred from the UPR Community Hospital to Tertiary Care Centers. During regular practice, the UPR Community Hospital calls ahead to ensure that space is available and inform the hospital service that the patient is being transferred. This process allows for efficient and safe transfer of patients from one care center to another. In the days after Hurricane Maria, due to the interruption of all communication channels, patients were transferred blindly. One EM physician noted, "When we would receive trauma patients, we had to send them to the level one without being able to formally call them... we would just, if an ambulance came in, we were like, 'please, thank you for unloading this patient and here's another one, please go to the other hospital.' It was really bad for them and us as well. It was very hectic."

\section{Reduced Coordination With Ambulance Services}

Another significant problem identified by respondents was the challenge of communicating with ambulance services. Respondents noted that even when they identified a patient that needed to be transferred, they were often unable to secure an ambulance to transfer the patient for various reasons. As one EM physician noted, "We couldn't communicate with the ambulance systems... you couldn't coordinate for a patient to be transferred through our ambulance. At the same time, how are you going to discharge somebody who has an amputation and needs treatment." Another problem was ambulance services refusing to transfer patients unless they first received cash payment. One EM physician noted, "A big thing would be our partnership with the ambulances. For it to be stronger and for us to have an ambulance with us, at least right there, 24 hours... [then] we don't have to wait until paramedics, because they're from private companies... it's very odd and they basically want $\$ 250$ up front instead of transferring the patient that's going to die in their face." The problem was that the hospital could not communicate to the ambulance services that the patient's insurance would cover ambulance, so private companies demanded cash payment. Another respondent noted, "Once we were able to move patients, we didn't have the transportation, the ambulances... while we worked to maintain the health and conditions of the patients, the ambulance companies didn't want to accept any medical plans."

\section{Poor Response Coordination With Government}

Many respondents noted poor and inefficient coordination with the Puerto Rican government. As one administrator noted, "When everything happened, communication with agencies was non-existing. We had to go to COE (Centro de Operacion de 
Emergencias, Emergency Operation Center), which was in San Juan. There, the government integrated all agencies into a single place, and that's how we all communicated. We had to go there to ask for help and determine what we were going to do." In the case of Hurricane Maria, vertical communication was absent or significantly slowed.

\section{Community Trust in Hospital and Healthcare System}

Respondents identified two themes that they felt contributed to more resilient response following Hurricane Maria. The first theme identified as contributing to resilience was the large degree of community trust in this PR Community Hospital in the weeks following Hurricane Maria. As one FM physician mentioned, "This hospital is known in the community as the 'Hospital of the area,' so the communities surrounding us know this is the hospital they go to when something happens. This is not just an emergency hospital, but a place the community comes for everything because they know this place provides all the services. The hospital published a group of messages in the newspapers to communicate that it was open and able to provide services. That's another way for the community to know they could come and would be assisted." The other theme was the relationship healthcare workers had cultivated with the community. This relationship aided resilience after the Hurricane and made patients confident they could rely on Puerto Rican health providers.

\section{Adaptive}

With regard to Adaptive, resilient hospitals and health systems are able to transform when under stress to meet the specific health needs of the population. Within Adaptive, we identified one domain as a resilience strength of the Puerto Rican health system.

\section{Organized Staffing Adjustments}

Respondents noted that the UPR Community Hospital staff was remarkably flexible in the days and weeks after the Hurricane. The UPR Community Hospital increased staff and work hours in the weeks following the Hurricane and the physicians were able to adapt without problem. As one EM physician states, "They did a really good job right after making sure that we had enough staff. We worked doubles. Not double shifts, but we had a double coverage. The nursing staff was really amazing about that. We have people that live really far away. We had one nurse who actually lost her whole house and they were really supportive about getting her new uniforms and helping her get to work. We rallied together. They were really helpful in that sense." Despite the difficulties in coordinating care and infrastructure, the Puerto Rican health care staff was able to effectively adapt to the circumstances in a manner that cultivated resilience.

\section{DISCUSSION}

It has been over 2 years since Hurricane Maria made landfall in Puerto Rico, and the impact of the Hurricane can still be felt today. Various studies have shown the widespread devastation that Hurricane Maria had on the people of Puerto Rico: taking nearly 5,000 lives, devastating the health system, and destroying the physical infrastructure of the island (Kishore et al., 2018). Our study focuses not on the magnitude of the destruction, but on the specific factors intrinsic to the Puerto Rican health system that may have predisposed the island's population to experiencing more severe health outcomes. Specifically, we performed a "critical case" case study guided by health system resilience framework using semistructured interviews with health care providers at a UPR Community Hospital. Figure 1 uses the Kruk framework of health system resilience to explain resilience in Puerto Rico following Hurricane Maria.

This study identified key factors within the Aware, SelfRegulated, and Integrated domains that were especially weak and were the key factors that led to a poorly resilient hospital and health system. The findings pointed to specific areas that can be targeted to improve the resilience of the Puerto Rican health system and can be adapted to other health systems. First, ensuring that key hospitals have adequate back-up generators is imperative. The lack of back-up generators resulted in an interruption of health services that spilled over into other domains of resilience. Puerto Rico and other nations with limited resources constantly facing threats of natural disasters must prioritize funding back-up generators at key hospitals.

Second, our study corroborated the findings of previous studies that the patients most at risk for adverse health outcomes immediately after a natural disaster are patients with chronic conditions. These patients rely on constant access to medications and health services for their livelihood. The most dramatic example of these patients is those with ESRD, but also included in this patient population are those with heart failure, chronic psychiatric conditions, and COPD patients. Following Hurricane Maria, the health system was overwhelmed by these patients presenting acutely decompensated conditions requiring emergency management. To ensure that these patients are adequately cared for, pharmacies and dialysis centers also need to be prioritized as healthcare facilities with adequate back-up generators and resources.

Third, there must be improved measures for communication both horizontally and vertically. Horizontally, a system must be put in place to transfer critically ill patients to appropriate facilities with back-up generators. Vertically, the government must have a system in place that ensures emergency medical services are guaranteed reimbursement during a natural disaster.

Our study did have limitations. Most significantly, this is a qualitative case study with a limited number of participants. Because this was a single-institution case study our findings may not be generalizable. However, the study was designed to sample a "critical case" scenario, and while our findings cannot be generalizable to all healthcare systems, a logical generalization can be made that, similar to the UPR Community Hospital, other PR hospitals logically may have had similar or worse experiences. In addition, although we interviewed a range of providers and administrators, we did reach data saturation and our respondents were able to speak not just to about the UPR Community Hospital but also to its interplay within Puerto Rico and the healthcare system as a whole. That being said, future resilience framework studies should incorporate the 
perspectives to health providers and other institutions and to public health professionals.

In September 2017, the people of Puerto Rico were devastated by Hurricane Maria. When there is a stress to a health system, the population will inevitably experience some heightened degree of social disruption, morbidity and mortality. As we have seen with the outbreak of COVID-19, it is as important as ever that both developed and developing countries are fortified with resilient health systems so that when an inevitable stress occurs, social disruption and excess morbidity can be minimized. Unfortunately, the degree of devastation experienced by the people of Puerto Rico after Hurricane Maria demonstrates a poorly resilient health system (Pullen, 2018; Roman, 2018). Yet, up to this point, there has not been a study that examines the specific aspects of the Puerto Rican health system that resulted in this poorly resilient response. Our critical case study is the first to identify aspects of the Puerto Rican health system that led to a poorly resilient response and can be targeted by the Puerto Rican Public Health officials to improve the resilience before the next disaster hits. With limited government funding and global warming resulting in more devastating storms each year, it is imperative that Puerto Rico and other resource limited nations focus their investments appropriately in order to cultivate health system resilience and protect their populations against the next inevitable health system shock.

Most importantly, conclusions from our critical case study supports the use of the Kruk et al. framework as a useful tool to evaluate health systems following events that cause stress in the system, such as natural disasters or pandemics. This finding is especially relevant as the COVID virus has taken over 200,000 lives in the United States and public health experts are searching for tools to scrutinize the preparation and response of the United States government. Currently, there is much debate about what specific factors in the health system may have predisposed the United States to a suboptimal response. Our study shows that the Kruk et al. health system resilience framework would be an appropriate tool to evaluate the response of the US health system and could direct future investments to ensure that the government is better prepared for future pandemics and natural disasters.

\section{REFERENCES}

Abimbola, S., and Topp, S. M. (2018). Adaptation with robustness: the case for clarity on the use of 'resilience' in health systems and global health. BMJ Glob. Health 3:e000758. doi: 10.1136/bmjgh-2018-000758

Blanchet, K., Nam, S. L., Ramalingam, B., and Pozo-Martin, F. (2017). Governance and capacity to manage resilience of health systems: towards a new conceptual framework. Int. J. Health Policy Manag. 6, 431-435. doi: 10.15171/ijhpm.2017.36

Dedoose, D.V. (2018). Web Application for Managing, Analyzing, and Presenting Qualitative and Mixed Method Research. Los Angeles, CA: SocioCultural Research Consultants.

Greenstein, J., Chacko, J., Ardolic, B., and Berwald, N. (2016). Impact of Hurricane sandy on the Staten Island university hospital emergency department. Prehosp. Disaster Med. 31, 335-339. doi: 10.1017/S1049023X16000261

Hakim, R. M., and Lazarus, J. M. (1995). Initiation of dialysis. J. Am. Soc. Nephrol. 6:1319.

\section{DATA AVAILABILITY STATEMENT}

The original contributions presented in the study are included in the article/supplementary materials, further inquiries can be directed to the corresponding author/s.

\section{AUTHOR CONTRIBUTIONS}

This project was carried out by CR as the topic of his Stanford Medical Scholars research project under the supervision of VJ. Details on the scope of the Stanford Medical Scholars Program can be found at https://med.stanford.edu/medscholars.html. CR was primarily responsible for designing the study, implementing the resilience framework, conducting the interviews, analyzing the data, and drafting the manuscript. EL provided input on adapting the Kruk resilience framework for this study. RR-G and JG assisted in translating the framework and identifying participants at the UPR Community Hospital. JB and MB were involved in data analysis and manuscript preparation. VJ was involved in the conception, design, organization of resources, data analysis, and manuscript preparation. VJ attests that all listed authors meet authorship criteria and that no others meeting the criteria have been omitted and confirms that the manuscript is an honest, accurate, and transparent account of the study being reported and that no important aspects of the study have been omitted. All authors contributed to the article and approved the submitted version.

\section{FUNDING}

This work was supported by an NIH R01 HL134776 and R01 HL139664 to VJ. CR was supported by a Stanford Medical Scholars Program.

\section{ACKNOWLEDGMENTS}

The authors would like to thank the staff of the UPR Community hospital for taking the time to participate in this study. The authors plan to report the results of this study to all participants.

Kishore, N., Marques, D., Mahmud, A., Kiang, M. V., Rodriguez, I., Fuller, A., et al. (2018). Mortality in Puerto Rico after Hurricane Maria. N. Engl. J. Med. 379, 162-170. doi: 10.1056/NEJMsa18 03972

Kruk, M. E., Ling, E. J., Bitton, A., Cammett, M., Cavanaugh, K., Chopra, M., et al. (2017). Building resilient health systems: a proposal for a resilience index. BMJ 357:j2323. doi: 10.1136/bmj.j2323

Kruk, M. E., Myers, M., Varpilah, S. T., and Dahn, B. T. (2015). What is a resilient health system? Lessons from Ebola. Lancet 385, 1910-1912. doi: 10.1016/S0140-6736(15)60755-3

Kutzin, J., and Sparkes, S. P. (2016). Health systems strengthening, universal health coverage, health security and resilience. Bull. World Health Organ. 94:2. doi: 10.2471/BLT.15.165050

Li, X., Huang, J., and Zhang, H. (2008). An analysis of hospital preparedness capacity for public health emergency in four regions of China: Beijing, Shandong, Guangxi, and Hainan. BMC Public Health 8:319. doi: $10.1186 / 1471-2458-8-319$ 
Lin, R. K. (1993). Applications of Case Study Research. Thousand Oaks, CA: SAGE Publications, the Univeristy of California,

Ling, E. J., Larson, E., Macauley, R. J., Kodl, Y., VanDeBogert, B., Baawo, S., et al. (2017). Beyond the crisis: did the Ebola epidemic improve resilience of Liberia's health system? Health Policy Plan. 32, iii40-iii47. doi: 10.1093/heapol/czx109

Mattei, J., Tamez, M., Rios-Bedoya, C. F., Xiao, R. S., Tucker, K. L., and RodriguezOrengo, J. F. (2018). Health conditions and lifestyle risk factors of adults living in Puerto Rico: a cross-sectional study. BMC Public Health 18:491. doi: 10.1186/s12889-018-5359-Z

Mokdad, A. H., Mensah, G. A., Posner, S. F., Reed, E., Simoes, E. J., and Engelgau, M. M. (2005). Chronic, and vulnerable populations in natural disasters working, When chronic conditions become acute: prevention and control of chronic diseases and adverse health outcomes during natural disasters. Prev. Chronic Dis. 2, 1-4.

O’Brien, B. C., Harris, I. B., Beckman, T. J., Reed, D. A., and Cook, D. A. (2014). Standards for reporting qualitative research: a synthesis of recommendations. Acad. Med. 89, 1245-1251. doi: 10.1097/ACM.00000000000 00388

Patton, M. Q. (2002). Qualitative Research and Evaluation Methods. Thousand Oaks, CA: SAGE.

Pullen, L. C. (2018). Puerto Rico after Hurricane Maria. Am. J. Transpl. 18, 283-284. doi: 10.1111/ajt.14647
Roman, J. (2018). Hurricane Maria: a preventable humanitarian and health care crisis unveiling the Puerto Rican dilemma. Ann. Am. Thorac. Soc. 15, 293-295. doi: 10.1513/AnnalsATS.201710-792OI

Santos-Burgoa, C., Goldman, A., Andrade, E. L., Barrett, N., Colón-Ramos, U., Edberg, M., et al. (2018). Ascertainment of the Estimated Excess Mortality from Hurricane Maria in Puerto Rico. Washington, DC: The George Washington University, Milken Institute School of Public Health.

Strauss, A., and Corbin, J. M. (1998). Basic of Qualitative Research: Techniques and Procedures for Developing Grounded Theory. Thousand Oaks, CA: SAGE Publications.

Conflict of Interest: The authors declare that the research was conducted in the absence of any commercial or financial relationships that could be construed as a potential conflict of interest.

Copyright (C) 2021 Rios, Ling, Rivera-Gutierrez, Gonzalez Sanchez, Merrell, Bruce, Barry and de Jesus Perez. This is an open-access article distributed under the terms of the Creative Commons Attribution License (CC BY). The use, distribution or reproduction in other forums is permitted, provided the original author(s) and the copyright owner(s) are credited and that the original publication in this journal is cited, in accordance with accepted academic practice. No use, distribution or reproduction is permitted which does not comply with these terms. 